\title{
Managing attention-deficit/hyperactivity disorder
}

\author{
Daniel A. Gorman MD, Elia Abi-Jaoude MD MSc
}

See also the Practice article by Jimenez and Guevara at www.cmaj.ca/lookup/doi/10.1503/cmaj.130042

\begin{abstract}
Before starting treatment for attention-deficit/hyperactivity disorder (ADHD), other potential causes for symptoms should be ruled out

Problems with focus and behaviour in some children may occur because of poor sleep, diet (e.g., food additives), hearing or vision impairment, environmental toxins (e.g., lead), learning difficulties, anxiety, depression or family dysfunction..$^{1-4}$ Symptoms may be developmentally appropriate, especially in young children with late birth dates who are compared with older classmates. ${ }^{5}$ Diagnostic and Statistical Manual of Mental Disorders criteria for ADHD should be met before treatment is considered.
\end{abstract}

Medications for ADHD are generally safe and well tolerated but can have adverse effects and are associated with some small but serious risks

The most common adverse effects are appetite suppression and insomnia with stimulants, sedation and upset stomach with atomoxetine, and mood changes and small increases in heart rate and blood pressure with both types of medication. ${ }^{910}$ Prolonged use of a stimulant typically causes modest growth suppression in children, use of atomoxetine is associated with a small risk of suicidality, and both treatments infrequently cause severe agitation or symptoms of mania or psychosis. ${ }^{2}$ Because very rare cases of sudden death have been reported in people taking these medications, patients should undergo screening for cardiac risk factors. ${ }^{11}$

\section{Nonpharmacological treatments can be effective and should generally be started first}

Controlled trials support the efficacy of behavioural therapy for children ${ }^{2}$ and cognitive-behavioural therapy for adults. ${ }^{6}$ Lifestyle modifications addressing diet, sleep and exercise may be helpful. ${ }^{7.8}$

Pharmacotherapy requires frequent monitoring and dose adjustments, especially during the initial titration phase

Stimulants should be started at a low dose and adjusted based on response and adverse effects; atomoxetine should be titrated as tolerated according to a weight-based algorithm. When a stimulant is used, either methylphenidate or an amphetamine can be started; if the response is poor, the other stimulant should be considered next. Monitoring includes evaluating changes in symptoms, functional impairment, adverse effects, body weight and (in children) height, and vital signs. ${ }^{2}$ Rating scales are available for tracking ADHD symptoms in children $^{2}$ and adults, ${ }^{12}$ including some that are available free of charge (e.g., www.adhd.net for children; www.hcp.med.harvard.edu/ncs/asrs .php for adults). Periodic drug-free trials (e.g., annually) should be considered to reassess the need for continued pharmacotherapy.
Pharmacotherapy may be appropriate for patients with severe impairment or with insufficient benefit from nonpharmacological treatments

Only stimulants (methylphenidate and amphetamines) and atomoxetine are approved by Health Canada for the treatment of ADHD in adults and children at least 6 years of age. Many placebo-controlled trials support the short-term efficacy of these agents for both age groups,,${ }^{9,10}$ but evidence for long-term benefit is far more modest. The advantages of stimulants over atomoxetine include rapid onset of action, higher response rate and greater effect size. Atomoxetine may be useful for patients with substance abuse issues, eating disorders or a history of poor responses or tolerability to multiple stimulants. $^{2}$

For references, see Appendix 1, available at www.cmaj.ca/lookup/suppl/doi:10.1503 /cmaj.121826/-/DC1

\section{Competing interests: None declared.}

This article has been peer reviewed.

Affiliations: Department of Psychiatry (Gorman, Abi-Jaoude), University of Toronto; Department of Psychiatry (Gorman), The Hospital for Sick Children; Department of Psychiatry (Abi-Jaoude), University Health Network, Toronto, Ont.

Correspondence to: Daniel A. Gorman, daniel .gorman@sickkids.ca, daniel.gorman@utoronto.ca

CMAJ 2013. DOI:10.1503/cmaj.121826 